\title{
Polyamine and Ethylene pathways crosstalk revisited on a genome wide and gene function scale
}

BLAISE PASCAL MUVUNYI ( $\sim$ muvunyi14@lzu.edu.cn )

Lanzhou University https://orcid.org/0000-0003-1244-5536

\section{Fan Wu}

Lanzhou University

Qi Yan

Lanzhou University

Gisele Kanzana

Lanzhou University

Yufei Zhang

Lanzhou University

Tiantian Ma

Lanzhou University

Xueyang Min

Lanzhou University

Zhuanzhuan Yan

Lanzhou University

Zhengshe Zhang

Lanzhou University

Yufeng Zhao

Lanzhou University

Yanrong Wang

Lanzhou University

Olivier Kamana

NIRDA

Jiyu Zhang

Lanzhou University

\section{Research article}

Keywords: Polyamine, ethylene, ABA, abiotic stresses, Cleistogenes songorica, Arabidopsis

Posted Date: July 9th, 2019 
DOl: https://doi.org/10.21203/rs.2.11103/v1

License: (c) (1) This work is licensed under a Creative Commons Attribution 4.0 International License. Read Full License 


\section{Abstract}

Background Polyamine and ethylene biosynthesis pathway genes are widely involved in the regulation of plant abiotic stresses. For their biosynthesis, both pathways require the same precursor, Synthase Adenosyl Methionine (SAM) enzyme. Whether they function as competitors or collaborators to regulate plant abiotic stress tolerance is still an elusive topic. Genome wide analysis of Cleistogenes songorica polyamine and ethylene pathway gene families was conducted to study their evolutionary relationship. And, using Arabidopsis plants transformed with a polyamine gene SAMDC2 from C. songorica, the expression of key genes from both pathways, and other previously well-studied stress responsive genes was investigated under salt or drought stress. Further, the ABA's role on this interaction salt stress was also studied. Results 17 polyamine, 12 ethylene and 6 SAM biosynthesis related genes were identified at genome wide level in C. songorica. Phylogenetic analysis revealed close evolutionary similarities between gene families from both pathways. Also, analysis of cis regulatory elements indicated that SAM family genes promoters were rich into both $A B A$ and ethylene related cis regulatory elements. Transcriptomic analysis, qRT-PCR validation, and confirmation using transgenic Arabidopsis showed that polyamine and ethylene key pathway genes can be concurrently expressed during abiotic stresses. Arabidopsis plants expressing a polyamine gene CsSAMDC2 driven by RD29A showed an improved drought and salt stress tolerance, and an increased expression of key polyamine and ethylene pathway genes. These plants maintained higher chlorophyll content and photosynthetic capacity. Morphological analysis of transgenic seedlings showed that leaves of these lines exhibited a more compact architecture following salt stress exposure. Application of ABA on transgenic lines under salt stress further improved the expression of polyamine and ethylene pathway genes. Further, lateral and primary root development were found improved during salt stress and ABA treatments. Interestingly, the expression of ethylene pathway genes was not reversed by exogenous ABA during salt stress treatment. Conclusion In silico and gene functional analysis assays revealed potential evolutionary and functional similarities between polyamine and ethylene pathway gene families. Such findings imply a synergetic interaction between polyamine and ethylene pathways, and the significant role of ABA on this crosstalk.

\section{Abstract}

Background: Polyamine and ethylene biosynthesis pathway genes are widely involved in the regulation of plant abiotic stresses. For their biosynthesis, both pathways require the same precursor, Synthase Adenosyl Methionine(SAM) enzyme. Whether they function as competitors or collaborators to regulate plant abiotic stress tolerance is still an elusive topic. Genome wide analysis of Cleistogenes songorica polyamine and ethylene pathway gene families was conducted to study their evolutionary relationship. And, using Arabidopsis plants transformed with a polyamine gene SAMDC2 from $C$. songorica, the expression of key genes from both pathways, and other previously well-studied stress responsive genes was investigated under salt or drought stress. Further, the ABA's role on this interaction salt stress was also studied. 
Results: 17 polyamine, 12 ethylene and 6 SAM biosynthesis related genes were identified at genome wide level in $C$. songorica. Phylogenetic analysis revealed close evolutionary similarities between gene families from both pathways. Also, analysis of cis regulatory elements indicated that SAM family genes promoters were rich into both $\mathrm{ABA}$ and ethylene related cis regulatory elements.

Transcriptomic analysis, qRT-PCR validation, and confirmation using transgenic Arabidopsis showed that polyamine and ethylene key pathway genes can be concurrently expressed during abiotic stresses. Arabidopsis plants expressing a polyamine gene CSSAMDC2 driven by RD29A showed an improved drought and salt stress tolerance, and an increased expression of key polyamine and ethylene pathway genes. These plants maintained higher chlorophyll content and photosynthetic capacity. Morphological analysis of transgenic seedlings showed that leaves of these lines exhibited a more compact architecture following salt stress exposure.

Application of $A B A$ on transgenic lines under salt stress further improved the expression of polyamine and ethylene pathway genes. Further, lateral and primary root development were found improved during salt stress and ABA treatments. Interestingly, the expression of ethylene pathway genes was not reversed by exogenous ABA during salt stress treatment.

Conclusion: In silico and gene functional analysis assays revealed potential evolutionary and functional similarities between polyamine and ethylene pathway gene families. Such findings imply a synergetic interaction between polyamine and ethylene pathways, and the significant role of ABA on this crosstalk.

Keywords: Polyamine, ethylene, ABA, abiotic stresses, Cleistogenes songorica, Arabidopsis

\section{Background}

Abiotic stresses such as droughts, heat waves and salt severely burden crop productivity, and are expected to exacerbate as a consequence of climate change [1]. During stress injuries, stress responsive genes play important defensive role by mobilizing diverse functional metabolites to stabilize cellular components, mitigate oxidative stresses, and maintain proper water status.

Polyamine are among these metabolites that alleviate cellular injuries during abiotic stresses [2] by improving the structure and stability of functional proteins and nucleic acid [3]. Also, they are known to control the expression of functional genes during various stresses[4]. The widely studied plant polyamines are putrescine, spermine and spermidine. Expression of genes encoding enzymes involved in the biosynthesis of these polyamines improved polyamine contents and stress tolerance of several crops [5]. For instance, overexpressing $O D C, A D C, S A M D C$ and $S P D S$ genes in rice [6], tomato [7] and tobacco [8] conferred a broad spectrum of abiotic stress tolerance in these plants. Moreover, various studies have documented an induction of $S A M D C$ gene transcripts in plants exposed to a range of stresses including salt, high temperature, drought, and oxidative stress. 
Plant response to drought and/or salt stresses largely depends on how they sense signals from these stresses [2], and signaling crossroads are common in stress responsive pathway [9]. The metabolic pathway for polyamine biosynthesis is interconnected with other stress regulators and phytohormones including ABA [10] and ethylene [11]. These hormones are key regulators of several physiological responses including biotic and abiotic stresses signaling cascades[12]. They also enhance the activity of transcriptional regulators in stress responsive genes[13].

The metabolic pathway for polyamine biosynthesis is interlinked with ethylene pathway through SAM that acts as a common substrate for both metabolic routes[11]. Right downstream of SAM, are SAMDC and $\mathrm{ACO}$ enzymes for polyamines and for ethylene biosynthesis pathways, respectively[14]. SAM is converted to ACC by 1-aminocyclopropane-1-carboxylate (ACC) synthase enzyme. Next, ACC is oxidized to ACO that is finally converted to ethylene[15]. The antagonistic nature of both pathways has been well documented $[16,17]$; while polyamines may retard fruit, flower and/or leaf senescence and ripening, ethylene would contribute to their occurrence[16]. And silencing ACC or ACO genes shifted the activity of SAM in favor of polyamine pathway[18]. Yet, a recent study indicated that $S A M$ gene can function as a signaling molecule for $A B A$ and $\mathrm{H}_{2} \mathrm{O}_{2}$, and Medicago truncatula plants expressing MFSAM gene exhibited an improved cold tolerance while maintaining stable levels of polyamine and ethylene biosynthesis related genes activities[19]. Furthermore, Abscisic Acid Responsive Element (ABRE) cis-regulatory elements were found in both polyamine (SAMDC) [20] and ethylene (ACO) family genes [21] , and it was also shown in different plant species that a short term saline shock induces both polyamines and ethylene levels [22].

Drought and salt tolerant plants such as xerophyte and halophytes have evolved with particular adaptation strategies. Cleistogenes songorica, a xerophyte C4 desert plant, is widely distributed in wild areas in the north west part of China where annual precipitations are around $100 \mathrm{~mm}$. It is known to have novel drought stress adaptation strategies and contains rich pools of stress tolerance genes [23]. Earlier studies have shown that transgenic Arabidopsis lines expressing $C$. songorica $L E A$ genes exhibited improved salt and drought tolerance by reducing $\mathrm{CO}_{2}$ assimilation levels [24]. Moreover, gene identification and transcriptome analysis in other xerophytes, such as Populus euphratica has allowed to functionally identify several stress responsive genes $[25,26]$.

Polyamine and ethylene pathway have intricate functions in the regulation of plant abiotic stress signaling mechanism $[27,28]$. How the two pathways collaborate to answer to the same ultimate functions, and the role played by ABA on their interaction is yet to be investigated by genome wide analysis of $C$. songorica's polyamine and ethylene pathway and functional analysis of a C.songorica polyamine gene into Arabidopsis.

\section{Materials And Methods}

Identification of $C$. songorica polyamine and ethylene pathways gene families 
Arabidopsis (Arabidopsis thaliana. L), rice (Oryza sativa. L) and maize (Zea mays. L) protein sequences of genes involved in polyamine and ethylene metabolic pathways were used as queries to Blast search against $C$. songorica reference genome available at BMK cloud platform http://www.biocloud.net/. To obtain protein sequences of Arabidopsis polyamine genes, genes accessions of the previously reported Arabidopsis polyamine genes [29] were used to search against Arabidopsis protein genome data retrieved from plant genomics database ( https://phytozome.jgi.doe.gov/pz/portal.html)[30]. Protein sequences of genes involved in ethylene pathway were obtained from previously published Arabidopsis ACO/20G(Fell) [31] and ACS genes families [32]. Oryza sativa, and Zea mays polyamine and ethylene SAM family gene accessions were obtained by querying gene identifiers against the respective plant genome data in phytozome[30]. The obtained accession numbers were then used to retrieve the respective protein sequences from their corresponding protein full genome sequence [30].

The putative $C$. songorica polyamine and ethylene pathway protein sequences were analyzed according to Pfam family using Hidden Markov Model [23] within Pfam database https://pfam.sanger.ac.uk/search[33]. The resulting $C$. songorica protein sequences were then submitted to SMART (https://smart.emblheidelberg.de/) [34] and to the conserved domain search within NCBI (https://www.ncbi.nlm.nih.gov/Structure/cdd/wrpsb.cgi) databases [35] to confirm the presence of ethylene and polyamine related pathway gene protein domains. The obtained proteins were then named based on their Pfam conserved protein domain families and the appellations adopted for $A$. thaliana, and their sequential order on different $C$. songorica chromosomes and contigs.

Multiple sequence alignment and phylogenetic analysis of $C$. songorica polyamine and ethylene proteins.

To assign polyamine and ethylene pathway proteins to their respective clades, $C$. songorica polyamine and ethylene pathway protein sequences were aligned with Arabidopsis and $O$. sativa polyamine and ethylene protein sequences. All the protein sequences with their respective accession numbers are shown in Additional file 6. The multiple proteins sequence alignment was performed by using ClustalW algorithm (Feng and Doolittle 1987) within MEGA 6 software. The resulting protein sequence alignments were then used to construct phylogenetic tree after pair-wise deletion of gaps with p-distance matrix [36]. The Neighbor-Joining algorithm was used with one thousand bootstrap [36, 37].

cis-regulatory element and motif analysis

C. songorica sequences of $2000 \mathrm{bp}$ of the gene promoter region corresponding with the identified polyamine and ethylene genes were analyzed for putative cis-regulatory elements using PlantCARE database (http://bioinformatics.psb.ugent.be/webtools/plantcare/htm//) [38]. We then recorded and compared the number of stress and hormonal-related cis-regulatory elements in individual genes from polyamine and ethylene pathway, and from SAM family.

Transcriptomic analyses and qRT-PCR validation 
C. songorica seeds were sown in vermiculite medium supplied with $1 / 4$ diluted Hoagland's nutrient solution, pH 5.8. Growth chamber conditions were set at $75-80 \%$ relative humidity, $30 / 28^{\circ} \mathrm{C}$ (day / night), and $16 / 8 \mathrm{~h}$ (day / night) at the light intensity of $200 \mathrm{mmol}$ photons $\mathrm{m}^{-2} \mathrm{~s}^{-1}$. For heat stress treatment, one-month old seedlings were treated at $40{ }^{\circ} \mathrm{C}$ for $24 \mathrm{~h}$. Dehydration stress treatment was done by withholding irrigation to $6-10 \%$ of soil water content (for mild drought stress), and to $1-3 \%$ for severe dehydration stress. For salt stress treatment, different $\mathrm{NaCl}$ concentrations $(50 \mathrm{mM} ; 100 \mathrm{mM} ; 200 \mathrm{mM})$ were prepared and applied on seedlings by spraying. In another set of experiment seedlings were treated with $100 \mu \mathrm{M}$ ABA to investigate the role of $A B A$ on polyamine and ethylene pathway genes expression. For each treatment, root and shoot tissue samples were collected at 0 and $24 \mathrm{~h}$ post treatment and kept at $-80^{\circ} \mathrm{C}$ till RNA extraction. Three samples were collected for each treatment. Total RNA was isolated from root or shoot samples using the Shengong RNA isolation kit as instructed (Shengong Ltd, Shanghai, China). The isolated RNA samples were further pooled, and sequenced following Illumina sequencing guidelines. The root and shoot total RNA samples were also used to synthesize first strand cDNAs using an oligo dT primer and the cDNA synthesis kit (Shengong). The cDNAs samples were individually diluted to $100 \mathrm{ng} / \mu \mathrm{L}$ prior to qPCR using gene specific primers (Additional file 2). For each PCR reaction, three biological replicates with three technical replicates each were used.

qPCR reaction was conducted using 40 cycles of $95^{\circ} \mathrm{C} 5 \mathrm{~s}, 60^{\circ} \mathrm{C} 15 \mathrm{~s}$, and $72^{\circ} \mathrm{C} 34 \mathrm{~s}$, a SYBR Green Master (Shengong), and gene specific primers. The relative gene expression levels were analyzed using the comparative $\triangle \triangle C$ t method [39]. The expression level of $C$. songorica GADPH gene was used as an internal control. Two ways analysis of variance and Duncan's multiple range test (DMRT) were used for multiple mean comparisons. SPSS (IBM Corp. 2013, IBM SPSS Statistics for Windows, Version 21.0, Armonk, NY) was used to determine the significant differences between means $(p<0.005)$.

Cloning and transformation of a polyamine gene, CSSAMDC2 into Arabidopsis

The amplification of cDNA CSSAMDC2 (EST) was performed using a SMARTTM RACE CDNA Amplification Kit (Clontech, Japan). After sequencing, both 5' and 3' ends primers (Supplementary Table 1) of CSSAMDC2 were designed and used to amplify CSSAMDC2 open reading frame. The PCR was conducted according user manual (Clontech, USA). The same primers were used for full length cDNA amplification of $2.1 \mathrm{~kb}$ of $C S S A M D C 2$ genomic sequence. The resulting PCR products were ligated into the binary vectorpB 121 under the control of a constitutive promoter, CaMV35S and stress inducible promoter $R D 29 A$, and sequenced at Shanghai Shenggong Biotechnological Ltd. (Shanghai, China). The vector harbored selectable marker nptll conferring kanamycin resistance. The recombinants plasmids were separately mobilized into Hypervirulent Agrobacterium tumefaciens, strain GV3101, before transformation into Arabidopsis using floral dipping method. The expression of CSSAMDC2 gene was assessed in T3 plants using real-time PCR and AtActin (AY096397) as a housekeeping gene.

Stress treatment of Arabidopsis transgenic plants 
Wild type and transgenic Arabidopsis were cultivated in a mixture of peat and vermiculite soils (2:1). Growth chamber conditions were set at $75-80 \%$ relative humidity, $22^{\circ} \mathrm{C}$, and $16 / 8 \mathrm{~h}$ (day / night) with the light intensity of $200 \mathrm{mmol}$ photons $\mathrm{m}^{-2} \mathrm{~s}^{-1}$. For drought tolerance study, irrigation was withheld until $1-3 \%$ of soil water content (SWC) for both three-week old wild-type and transgenic plants.

For salt stress tolerance analysis, germination percentage of transgenic and wild-type was assayed by growing wild-type and transgenic lines on MS plates containing different $\mathrm{NaCl}$ concentration $(0 \mathrm{mM}$; $100 \mathrm{mM} ; 150 \mathrm{mM}$ ). In another set of experiments of salt stress study, plants were grown on MS medium for seven days before being transferred on new MS plates containing 100 or $150 \mathrm{mM} \mathrm{NaCl}$ supplemented or not with $100 \mu \mathrm{M}$ ABA. For each plant, the whole plant was sampled at 0 and $24 \mathrm{~h}$ for further physiological assays and gene expression analysis. Phenotypes of both treated and untreated were photographed.

Expression analysis of polyamine and ethylene pathway genes under different stress treatments in transgenic Arabidopsis

Drought, salt, and salt +ABA treatments were carried out to compare the expression levels of key polyamines pathway genes (AtADC2, AT4G34710; AtSAMDC1, AT3G02470; AtSPMS, AT5G53120and ethylene genes (ACC synthase AT5G51690; ACC oxidase, AAC27484) in transgenic and wild-type Arabidopsis plants.

Measurement of chlorophyll content

Chlorophyll content was determined according to Arnon[40]. For each sample leaf tissues weighing $100 \mathrm{mg}$ were detached and bleached by soaking them in $10 \mathrm{ml}$ of $30 \mathrm{~mL} 80 \%(\mathrm{~V} / \mathrm{V})$ ethanol. The mixture was incubated under dark for 24 hours then centrifuged for $5 \mathrm{~min}$ at 13,000 rpm using micro centrifuge (Eppendorf MiniSpin ${ }^{\circledR}$ ) at room temperature to fully remove leaf pigmentation. The optical density of the mixture was measured at $654 \mathrm{~nm}$ using a spectrophotometer.

Chlorophyll Fluorescence Fv/Fm

The quantum efficiency of PSII is represented by dark-adapted values given by the ratio Fv/Fm. This parameter is considered as a key indicator of plant photosynthetic performance [41]. Lower values reflect damage to the PS II complexes, and thus certain level of photo inhibition [42]. The values of this parameter were assayed between transgenic and wild-type plants. Three plant samples per line were primarily incubated for 30 minutes under dark conditions, and values were recorded for each individual line considering three replications per treatment using a PAM-2100 (Heinz Walz GmbH). The determination of maximum quantum yield PSIl was by using the formula Fv/Fm $=(\mathrm{Fm}-\mathrm{Fo}) / \mathrm{Fm}$ Where: $\mathrm{Fm}$ is the maximum fluorescence yield. Fo, the yield of fluorescence in the absence of an actinic (photosynthetic) light; minimum fluorescence yield [43].

\section{Results}


A phylogeny analysis was conducted to breakdown the evolutionary relationship between gene families from polyamine and ethylene pathways. Results showed that clusters were formed based on gene families not on metabolic pathways (Fig.1). Ethylene pathway clades of genes could be found adjacent to polyamines pathway gene clusters. For example, $S A M D C$ family of genes (polyamine pathway) was found grouped in the same cluster with ACS gene family (ethylene pathway), while SAM family genes were cladded next to both polyamine and ethylene pathway gene clusters

Promoter regions of SAM family genes are rich into stress related cis regulatory elements

Promoter regions of stress responsive genes are generally rich into cis regulatory elements that provide binding sites for transcriptions factors $[44,45]$. Herein, we surveyed the type and amount of stress related cis regulatory elements in $C$. songorica polyamine and ethylene pathway genes, and in SAM family gene promoters to get insights on the contribution of these cis elements on the particular biological and physiological functions in each pathway. A plethora of CAAT and TATA enhancers was found in both pathways (Table 1). Strikingly, the average number of MYB and ABRE cis-regulatory elements was almost comparable in both polyamine and ethylene pathway gene promoter regions, with a slight difference in favor of polyamine genes. In average, 1.3 ABRE and $2 \mathrm{MYB}$ cis acting elements were counted in the promoter regions of polyamine pathway genes, while $1 \mathrm{ABRE}$ and 1.4 MYB were recorded for ethylene pathway genes. Promoters of SAM family genes were the most enriched in ABRE cis regulatory elements and averaged of 3.1 counts per gene. Also, higher number of G-box, W-box and Jasmonic acid inducible motifs, CGTCA-motif and TGACG-motif was recorded in the promoter regions of SAM family genes.

Transcriptomic analysis

Expression analysis revealed that many of $S A M$ family genes were constitutively expressed across normal irrigations, mild drought stress and high dehydration stress treatments (Fig. 2A). CSSAM1 and CsSAM5 gene expression levels were induced after dehydration treatments in both root and shoot tissues, and consequently their expression was reduced after re-watering. The expression level of CSSAM6 gene was consistently high across all the treatments, and re-watering remarkably reduced its expression particularly in root.

Conversely, expression levels of CSADC3, CSSAMDC2 and CSSAMDC3 (polyamine pathway genes) were remarkably induced under severe dehydration stress and sharply reduced after re-watering, in both roots and shoots tissues for CSADC3 and CSSAMDC3 genes. The expression level of CSSAMDC2 gene was the most induced by dehydration stress and its expression declined in shoot after re-watering.

Transcriptomic expression levels of Cs2OG(Fell)1, Cs2OG(Fell) 5 and Cs2OG(Fell)7 (ethylene pathway genes) increased in shoot after strong dehydration stress treatment, and re-watering reduced the expression of CsCs2OG(Fell)5 and Cs2OG(Fell)7 genes in shoot, while the expression level of Cs2OG(Fell) 1 gene was reduced in both tissues. The expression level of another ethylene pathway gene 
CSACC4 went up steadily with increasing dehydration stress and reduced after re-watering in both tissues (Fig. 2A).

Expression levels of polyamine and ethylene pathway genes were also analyzed under different levels of salt stress, heat stress, low temperature stress, and under ABA treatment (Fig. 2B). For ethylene pathway, the expression level of Cs2OG(Fell) 1 gene increased after applying $200 \mathrm{mM} \mathrm{NaCl}$ stress conditions, interestingly, ABA treatment did not reverse its expression in shoot. Similarly, the expression levels of Cs20G(Fell)3 gene increased in both tissues following $100 \mathrm{mM} \mathrm{NaCl}$, heat and ABA treatments. Also, the expression levels of another ethylene pathway gene CSACC1 were induced by $50 \mathrm{mM}$ of $\mathrm{NaCl}$ and low temperature treatment in shoot and root tissues, and its expression went up in root after ABA treatment. In the same metabolic pathway, CsACC3 gene exhibited an increased expression in both tissues after 100 $\mathrm{mM} \mathrm{NaCl}$ treatment, and its expression was also high in root after $200 \mathrm{mM} \mathrm{NaCl}$ stress conditions. Also, ABA treatment induced its expression level in shoot compared to normal treatment conditions.

For polyamine pathway genes, the relative expression level of $C S A D C 2$ gene was slightly induced by 100 $\mathrm{mM} \mathrm{NaCl}$ stress in roots and by ABA treatment in shoots. For SAMDC family genes, CSSAMDC4 gene expression was induced by all the treatments in shoot tissue. CSSPMS genes were constitutively expressed across all treatments in both tissues, and ABA slightly increased the expression levels of CSSPMS1, CSSPMS3 and CSSPMS4. The expression level of CSSPMS2 was highly induced in roots after $100 \mathrm{mM} \mathrm{NaCl}$ or $200 \mathrm{mM} \mathrm{NaCl}$ and heat treatments in both tissues. On the other hand, expression levels of SAM family genes were generally higher compared to other families in both pathways, and from all the analyzed genes, CSSAM6 gene exhibited the highest expression level in shoot tissues after ABA treatment (Fig. 2B)

qRT- PCR validation

Quantitative RT-PCR validations of these expression profiles were conducted for drought, salt stress and ABA treatments in root and shoot tissues (Fig. 3). For genes involved in ethylene biosynthesis pathway, CsACC3 was selected as a representative (Fig. 3A). qRT-PCR validated the expression of CsACC3 as it was induced by 16.87 times in shoot after strong dehydration stress, while its expression in root increased from 2.7 folds after $50 \mathrm{mM} \mathrm{NaCl}$ to 5.4 folds under $100 \mathrm{Mm} \mathrm{NaCl}$ treatment, and then dropped to 2.6 folds at $200 \mathrm{mM} \mathrm{NaCl}$. Treatment with ABA did not reduce its expression levels relative to control conditions. This trend correlates well with the findings from the transcriptomic analysis.

The expression of CSSAM6 gene, was induced by all levels of drought and salt treatment applied, more importantly, as observed during transcriptome analysis, its expression level was the highest in shoot after ABA treatment as it soared by 18.3 folds (Fig. 3B). The expression CsSAMDC2 was induced by all stress treatments and $A B A$ application. The expression level of CSSAMDC2 gene after strong dehydration stress in shoot was the highest as it increased by 754 times relative to control. Also, its expression was induced to 11.7 and 16.4 times in root and shoot tissues following ABA treatment (Fig. 3C).

Expression of CSSAMDC2 gene enhanced drought stress tolerance in transgenic Arabidopsis 
As CSSAMDC2 gene was highly induced in C. songorica under drought conditions, it was transformed into Arabidopsis under a stress responsive and constitutive promoter, RD29A and 35S, respectively. Using primers of CSSAMDC2 gene promoters, and transformed Arabidopsis genomic DNA, an amplicon band of around 1200 bp size was detected in 9 Arabidopsis lines (Additional file 1). Similarly, successful transformation of RD 29A and 35S promoters was also confirmed through PCR using Arabidopsis genomic DNA and gene specific primers (Additional file 2). Among these confirmed transgenic plants, two lines transformed with RD29A:: CSSAMDC2 gene construct (TL01 and TL18) and other two lines (TL05 and TL11) transformed with 35S:: CSSAMDC2 gene construct were selected for further abiotic stress analysis. The expression level of CSSAMDC2 gene was induced in the studied transgenic plants after 14 days of drought stress (Fig. 4A). CSSAMDC2 gene enhanced dehydration tolerance of transgenic Arabidopsis as compared to wild-type plants which showed signs of wilting after 14 days of water withdrawing (Fig 4B). Furthermore, CSSAMDC2 gene expression was found higher in lines harboring RD29A::CSSAMDC2 gene construct than in 35Cs::CsSAMDC2 transgenic plants. The expression of CSSAMDC2 gene was also followed by an improved chlorophyll content (Fig. 5A) and photosynthetic capacity (Fig. 5B) in transgenic Arabidopsis compared to wild-type plants.

CSSAMDC2 gene improved seed germination percentage in transgenic Arabidopsis under salt stress

Seed germination percentage of transgenic plants and wild-type plants was investigated on different concentrations of $\mathrm{NaCl}$. Under $0 \mathrm{mM} \mathrm{NaCl}$, germination rates of $98-100 \%$ were reached after 3 days following sowing. There was no significant difference among the studied lines (Additional file $3 \mathrm{~A}$ and $\mathrm{C}$ ). Under $100 \mathrm{mM} \mathrm{NaCl}$, germination percentage after nine days of salt stress exposure was $96 \%$ (TL01), 99\% (TL18); 97\% (TL05), and 98\% (TL11), while under the same stress conditions, it was at $84 \%$ in wildtype plants (Additional file 3B and D)

Phenotypes of Arabidopsis seedlings after extended exposure on salt stress

Wild-type Arabidopsis and transgenic lines expressing CSSAMDC2 gene were grown on $150 \mathrm{mM} \mathrm{NaCl}$ stress medium. After reaching maximum germination rate (after 28 days) (Additional file 4A), all seedlings were maintained under the same treatment conditions for18 days (Fig. 6A). The number of cotyledons that maintained green colors after treatment was found higher in transgenic seedlings than in their wild-type plants (Fig. 6B). Moreover, leaves of transgenic Arabidopsis showed a more compact architecture (Fig. 6A), a typical salt avoidance mechanism in stress tolerant plants[46, 47].

Expression of Polyamine and Ethylene pathway genes and other stress responsive genes under different treatments

The expression of different stress responsive genes including polyamine related genes AtSAMDC2 and $A t A D C 2$, and ethylene pathway genes, AtACO2 and AtACS6, an Ethylene responsive gene AtERF1, and ABA responsive gene AtRD29A were more improved in transgenic Arabidopsis after drought stress treatment than in their wild-type counterparts (Fig. 7A). 
All studied polyamine and ethylene pathway genes showed positive upregulations after salt stress (Fig. 7B) or salt stress $+A B A$ treatments (Fig. 7C). Analysis of the expression of key polyamine pathway genes AtSAMDC2 and $A t A D C 2$ under salt stress +ABA treatments revealed that their expression levels increased by 24 (AtSAMDC2) and 21 (AtADC2) folds in TL01, and 29 (AtSAMDC2) and 28.7(AtADC2) folds in TL18. (Fig. 7C). However, the expression of both genes only increased by 4.9 (AtADC2) and 4.7 folds (AtSAMDC2), respectively, in wild-type plants relative to non-treatment conditions. The activity of AtRD29A was also highly induced by salt stress (Fig. 7B), and ABA application enhanced its expression levels to 89 and 87 folds in TL01 and TL018 plants (Fig. 7C) respectively, which were the highest recorded expression levels in all lines analyzed.

Exogenous ABA altered plant architecture of transgenic Arabidopsis under salt tolerance

Analysis of leaves and root morphology in wild-type and transgenic lines grown on MS plates supplemented with $150 \mathrm{mM} \mathrm{NaCl}$ indicated no significant phenotypical differences between these lines after $72 \mathrm{~h}$ (Fig. 8B). However, application of ABA induced formation of lateral roots and triggered root growth in all lines compared to salt stress treatment alone (Fig. 8D). RD29A.:CsSAMDC2 lines exhibited the most improved lateral root development under these treatments conditions (Fig. 8D). Measurements of root length in RD29A::CSSAMDC2 lines of $4.3 \mathrm{~cm}$ (TL01) and $4.2 \mathrm{~cm}$ (TL018) were the highest, and were significantly different with those in other lines (Additional file 5B)

\section{Discussion}

Cleistogenes songorica is perennial monocots plants highly adapted to arid conditions. This study indicated that $S A M D C 2$ gene expressed in Cleistogenes songorica shoot and root tissues after high dehydration stress, can also enhance abiotic stress tolerance of Arabidopsis by inducing the expression of polyamines, ethylene and ABA stress responsive genes.

One of the strategies for abiotic stress tolerance in perennial monocots plants are the enhancement of photosynthesis and redox metabolisms, and tendency to form molecular chaperons [48]. Transgenic Arabidopsis plants expressing CSSAMDC2 gene showed an improved tolerance to salt and drought stresses, and higher photosynthetic parameters such a high Fv/Fm and chlorophyll content. Maintaining low chlorophyll degradation and high photosynthetic capacity is an imperative adaptation mechanism in stress tolerant species [49].

Different studies have previously shown that polyamine and ethylene compete for the same precursor Sadenosylmethionine (SAM), a donor of methyl group required by both metabolic pathways[50, 51]. The present study suggests that under stress conditions both pathways may enter a mutually beneficial synergistic relationship instead of merely competing for SAM. And for the first time, this hypothesis was studied on genome wide scale. Results from phylogenetic analysis indicated close evolutionary relationships between some gene families from polyamine and ethylene pathways. These findings may infer functional similarities that could have narrowed divergence of these families during their evolutionary history. A such gene functional evolution concept corroborates with former studies in 
Brassica napus $L E A$ gene family [52]. Also, SAM genes were found to be rich into cis acting elements which provide binding sites for both ethylene, Jasmonic and ABA related transcription factors. This could explain their functional relevance in mediating both polyamine and ethylene pathways during stresses conditions. The above results were further supported by a simultaneous increased expression of both polyamine and ethylene pathway genes, ethylene and ABA responsive gene in Arabidopsis expressing a polyamine gene CSSAMD2 during salt or drought stresses.

Other earlier studies suggested that instead of metabolically competing for $S A M$, other molecular mechanisms may play into their role $[53,54]$. For instance, it was proposed that the activity of AtERF1 gene enhanced plant tolerance to a broad spectrum of abiotic stresses by integrating Abscisic acid, ethylene and Jasmonic acid [55]. To investigate this hypothesis, exogenous ABA was applied on saltstressed transgenic lines and wild type plants. Results showed that during salt stress, application of $A B A$ further enhanced mRNA expression levels for polyamine (AtSAMDC2 and AtADC2) and ethylene pathway genes (AtACO2 and AtACS6), and for ABA (AtRD29A) and ethylene (AtERF1) responsive genes in transgenic Arabidopsis under salt stress. Interestingly, the expression of AtERF1 was found higher in RD29A::CSSAMDC2 lines than in 35S:::CSSAMDC2 andwild-type plants.

Furthermore, ABA predominantly promoted the expression of AtRD29A and AtSAMDC2 in RD29A::CsSAMDC2 Arabidopsis lines; TL01 and TL018. Subsequently, TL01 and TL018 showed improved primary and lateral and root growth. Previous studies showed that ABA induces lateral roots development under salt stress $[56,57]$ through its signaling that are transduced in cell specific manner and through intricate hormonal interactions $[58,59]$. However, some previous studies reported that lateral and primary root growth suppress each other's development[58]. Interestingly, in this study both traits were found enhanced in Arabidopsis plants expressing RD29A::CSSAMDC2 gene construct. This was possibly due to an enhanced activity polyamines genes, AtADC2 and AtSAMDC2. Polyamines are known to promote cell division and differentiation necessary for root apex and lateral root development [60]. It was previously shown that disrupting the activity of $A D C$ genes in Arabidopsis[61] or in P. vulgaris [62] correlated with reduced lateral root formation and primary root growth, respectively. Similarly, downregulating $S A M D C$ gene in potato plants was associated with limited root growth [63].

This study showed that though CSSAMDC2 may suppress salt stress under ABA independent manner, exogenous ABA could promote its activity by enhancing the upregulation of polyamine (AtADC2 and AtSAMDC2), ethylene (AtERF1, AtACO2, AtACO6) and ABA (AtRD29A) related stress responsive genes. From these findings we speculate that under abiotic stress $A B A$, ethylene and polyamine could mutually interact to respond to their respective functions in plant defensive mechanism. Moreover, as a results of a rich diversity cis acting elements found in the promoter regions of SAM family genes, we speculate that SAM enzymes may function as mediator for polyamine and ethylene pathways during stress conditions. A network for stress response regulation by polyamine, ethylene pathway genes through SAM was proposed in this study (Fig. 9), however further molecular assays are required for confirmation.

\section{Conclusions}


Phylogenetic and cis-regulatory analyses revealed potential evolutionary and functional similarities between polyamine and ethylene pathway gene families. Subsequently, the expression of genes from both pathways was induced during drought or salt stresses, or salt stress +ABA treatments in Arabidopsis expression a polyamine gene, CSSAMDC2. Findings from this study suggest molecular interaction between polyamine and ethylene pathways genes during salt or drought stresses, and the important role of $A B A$ on this crosstalk during salt stress.

\section{Abbreviations}

ABA: Abscisic Acid ABRE: Abscisic Acid Responsive Element ACC: 1-aminocyclopropane-1-carboxylate. ACO: 1-aminocyclopropane-1-carboxylate oxidase. ACS: 1-aminocyclopropane-1-carboxylate synthase. ADC: arginine decarboxylase. CaMV: Cauliflower Mosaic Virus. CDS: coding sequence. ERF: Ethylene responsive factor. EST: expressed sequence tag.

ODC: ornithine decarboxylase. MYB:myeloblastosis. 2OG(Fell): 2-oxoglutarate (2OG) and Fe(II). NaCl: Sodium Chloride. RNA: Ribonucleic acid. Put: putrescin. PSII: Photosystem II. SAM: Sadenosylmethionine. SAMDC. S-adenosylmethionine decarboxylase. SE: standard error. Spd: spermidine. SPDS: spermidine synthase. Spm: spermine. SPMS: spermine synthase. TL: transgenic line. qRTPCR: quantitative polymerase chain reaction. UORF: upstream open reading frame. UTR: untranslated region. V/V: volume/volume. WT: wild type.

\section{Declarations}

Ethics approval and consent to participate:

Not applicable.

Consent for Publication:

Not applicable.

Availability of data and material:

Most of the supporting data are presented in the additional file and plant materials are available at Lanzhou University in the laboratory of the Corresponding author (zhangjy@lzu.edu.cn). Information on Cleistogenes songorica genome sequencing are available at http://www.biocloud.net/ and can be accessed through the corresponding author.

Competing interests:

The authors declare that they have no competing interests. 
Funding: This work was supported by the Program for Changjiang Scholars and Innovative Research Team in University (IRT_17R50), the National Natural Science Foundation of China (31572453), the Open Project Program of State Key Laboratory of Grassland Agro-ecosystems (SKLGAE201702) and the 111 project (B12002).

Authors' contributions:

YW, JZ and BPM conceived the study. BPM and ZZ performed Arabidopsis transformation. ZZY and BPM conducted stress treatment analysis. BPM, QY, ZYY and WF contributed on RNA library preparing and sequencing. XM, BPM and GK conducted bioinformatics analysis. KO and TM curated data. BPM and YZ wrote the original draft and J.Z, BPM, KO reviewed and edited the manuscript. YW and JZ administered the project.

Acknowledgements: We thank Xin Shun Ding for his suggestions during manuscript preparation. We sincerely thank Zhen Duan for all the support she offered to complete this study. We thank the anonymous reviewers for their critical comments and detailed suggestions for revision

\section{References}

1. Fedoroff NV, Battisti DS, Beachy RN, Cooper PJ, Fischhoff DA, Hodges C, Knauf VC, Lobell D, Mazur BJ, Molden D: Radically rethinking agriculture for the 21st century. Science (New York, NY) 2010, 327(5967):833-834.

2. Liu J-H, Wang W, Wu H, Gong X, Moriguchi T: Polyamines function in stress tolerance: from synthesis to regulation. Frontiers in Plant Science 2015, 6:827.

3. Park MH: The post-translational synthesis of a polyamine-derived amino acid, hypusine, in the eukaryotic translation initiation factor 5A (elF5A). Journal of biochemistry 2006, 139(2):161-169.

4. Alet A, H Sánchez D, C Cuevas J, Marina M, Carrasco P, Altabella T, F Tiburcio A, Ruiz O: New insights into the role of spermine in Arabidopsis thaliana under long-term salt stress, vol. 182; 2012.

5. Minocha R, Majumdar R, Minocha SC: Polyamines and abiotic stress in plants: a complex relationship1. Frontiers in plant science 2014, 5:175.

6. Roy M, Wu R: Overexpression of S -adenosylmethionine decarboxylase gene in rice increases polyamine level and enhances sodium chloride-stress tolerance. Plant Science 2002, 163(5):987-992.

7. Cheng L, Zou Y, Ding S, Zhang J, Yu X, Cao J, Lu G: Polyamine accumulation in transgenic tomato

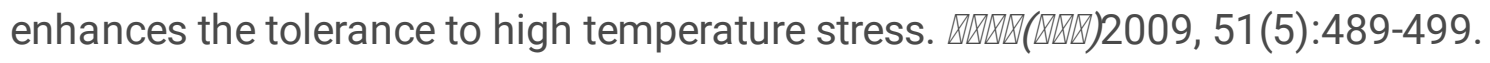

8. Waie B, Rajam MV: Effect of increased polyamine biosynthesis on stress responses in transgenic tobacco by introduction of human S -adenosylmethionine gene. Plant Science 2003, 164(5):727-734. 
9. Pál M, Szalai G, Janda T: Speculation: polyamines are important in abiotic stress signaling. Plant Science 2015, 237:16-23.

10. Cuevas JC, López-Cobollo R, Alcázar R, Zarza X, Koncz C, Altabella T, Salinas J, Tiburcio AF, Ferrando $A$ : Putrescine as a signal to modulate the indispensable ABA increase under cold stress. Plant Signaling \& Behavior 2009, 4(3):219-220.

11. Pandey S, Ranade SA, Nagar PK, Kumar N: Role of polyamines and ethylene as modulators of plant senescence. Journal of Biosciences 2000, 25(3):291-299.

12. Gerashchenkov G, Rozhnova N: The involvement of phytohormones in the plant sex regulation. Russian journal of plant physiology 2013, 60(5):597-610.

13. Forcat S, Bennett MH, Mansfield JW, Grant MR: A rapid and robust method for simultaneously measuring changes in the phytohormones $A B A, J A$ and $S A$ in plants following biotic and abiotic stress. Plant Methods 2008, 4(1):16.

14. Urano K, Yoshiba Y, Nanjo T, Igarashi Y, Seki M, Sekiguchi F, Yamaguchi-Shinozaki K, Shinozaki K: Characterization of Arabidopsis genes involved in biosynthesis of polyamines in abiotic stress responses and developmental stages. Plant Cell \& Environment 2003, 26(11):1917-1926.

15. Hedden P, Phillips AL: Manipulation of hormone biosynthetic genes in transgenic plants. Current Opinion in Biotechnology 2000, 11(2):130-137.

16. Bitrián M, Zarza X, Altabella T, Tiburcio AF, Alcázar R: Polyamines under Abiotic Stress: Metabolic Crossroads and Hormonal Crosstalks in Plants. Metabolites 2012, 2(3):516-528.

17. And PTE, Malmberg RL: Do Polyamines Have Roles in Plant Development? Annual Review of Plant Biology 1989, 40(1):235-269.

18. Wi SJ, Park KY: Antisense expression of carnation cDNA encoding ACC synthase or ACC oxidase enhances polyamine content and abiotic stress tolerance in transgenic tobacco plants. Molecules \& Cells 2002, 13(2):209-220.

19. Guo Z, Tan J, Zhuo C, Wang C, Xiang B, Wang Z: Abscisic acid, H2O2 and nitric oxide interactions mediated cold-induced S-adenosylmethionine synthetase in Medicago sativa subsp. falcata that confers cold tolerance through up-regulating polyamine oxidation. Plant Biotechnology Journal 2014, 12(5):601612.

20. Basu S, Roychoudhury A, Sengupta DN: Deciphering the Role of various cis-acting regulatory elements in controlling SamDC gene expression in Rice. Plant Signal Behav 2014, 9(3):-.

21. Wang Y-M, Yang Q, Liu Y-J, Yang H-L: Molecular Evolution and Expression Divergence of the Aconitase (ACO) Gene Family in Land Plants. Frontiers in Plant Science 2016, 7(1879). 
22. Zapata PJ, Serrano M, García-Legaz MF, Pretel MT, Botella MA: Short Term Effect of Salt Shock on Ethylene and Polyamines Depends on Plant Salt Sensitivity. Frontiers in Plant Science 2017, 8(855).

23. Zhang J, John UP, Wang Y, Li X, Gunawardana D, Polotnianka RM, Spangenberg GC, Nan Z: Targeted mining of drought stress-responsive genes from EST resources in Cleistogenes songorica. Journal of Plant Physiology 2011, 168(15):1844-1851.

24. Zhang J, Duan Z, Zhang D, Zhang J, Di H, Wu F, Wang Y: Co-transforming bar and CsLEA enhanced tolerance to drought and salt stress in transgenic alfalfa (Medicago sativa L.). Biochem Biophys Res Commun 2016, 472(1):75-82.

25. Qiu Q, Ma T, Hu Q, Liu B, Wu Y, Zhou H, Wang Q, Wang J, Liu J: Genome-scale transcriptome analysis of the desert poplar, Populus euphratica. Tree physiology 2011, 31(4):452-461.

26. Shi Y, Yan X, Zhao P, Yin H, Zhao X, Xiao H, Li X, Chen G, Ma X-F: Transcriptomic analysis of a tertiary relict plant, extreme xerophyte Reaumuria soongorica to identify genes related to drought adaptation. PLoS One 2013, 8(5):e63993.

27. Cao WH, Liu J, He XJ, Mu RL, Zhou HL, Chen SY, Zhang JS: Modulation of Ethylene Responses Affects Plant Salt-Stress Responses. Plant Physiology 2007, 143(2):707-719.

28. Shen $X$, Wang Z, Song X, Xu J, Jiang C, Zhao Y, Ma C, Zhang H: Transcriptomic profiling revealed an important role of cell wall remodeling and ethylene signaling pathway during salt acclimation in Arabidopsis. Plant Molecular Biology 2014, 86(3):303-317.

29. Alcázar R, F. Tiburcio A: Polyamines: Methods and Protocols; 2018.

30. Goodstein DM, Shu S, Howson R, Neupane R, Hayes RD, Fazo J, Mitros T, Dirks W, Hellsten U, Putnam $\mathrm{N}$ et al: Phytozome: a comparative platform for green plant genomics. Nucleic Acids Research 2012, 40(Database issue):D1178-D1186.

31. Ruduś I, Sasiak M, Kępczyński J: Regulation of ethylene biosynthesis at the level of 1aminocyclopropane-1-carboxylate oxidase ( ACO ) gene. Acta Physiologiae Plantarum 2013, 35(2):295307.

32. Yamagami T, Tsuchisaka A, Yamada K, Haddon WF, Harden LA, Theologis A: Biochemical Diversity among the 1-Amino-cyclopropane-1-Carboxylate Synthase Isozymes Encoded by the Arabidopsis Gene Family. Journal of Biological Chemistry 2003, 278(49):49102-49112.

33. Finn RD, Bateman A, Clements J, Coggill P, Eberhardt RY, Eddy SR, Heger A, Hetherington K, Holm L, Mistry J et al: Pfam: the protein families database. Nucleic Acids Research 2014, 42(Database issue):D222-D230. 
34. Letunic I, Copley RR, Schmidt S, Ciccarelli FD, Doerks T, Schultz J, Ponting CP, Bork P: SMART 4.0: towards genomic data integration. Nucleic Acids Res 2004, 32(Database issue):D142-144.

35. Marchler-Bauer A, Derbyshire MK, Gonzales NR, Lu S, Chitsaz F, Geer LY, Geer RC, He J, Gwadz M, Hurwitz DI et al: CDD: NCBI's conserved domain database. Nucleic Acids Res 2015, 43(Database issue):D222-226.

36. Tamura K, Stecher G, Peterson D, Filipski A, Kumar S: MEGA6: Molecular Evolutionary Genetics Analysis version 6.0. Mol Biol Evol 2013, 30(12):2725-2729.

37. Higgins DG, Sharp PM: CLUSTAL: a package for performing multiple sequence alignment on a microcomputer. Gene 1988, 73(1):237-244.

38. Rombauts S, Dehais $\mathrm{P}$, Van Montagu M, Rouze P: PlantCARE, a plant cis-acting regulatory element database. Nucleic Acids Res 1999, 27(1):295-296.

39. Fujisawa M, Takita E, Harada H, Sakurai N, Suzuki H, Ohyama K, Shibata D, Misawa N: Pathway engineering of Brassica napus seeds using multiple key enzyme genes involved in ketocarotenoid formation. Journal of Experimental Botany 2009, 60(4):1319-1332.

40. Arnon DI: Copper enzymes in isolated chloroplasts. Polyphenoloxidase in Beta vulgaris. Plant physiology 1949, 24(1):1.

41. Krause GH, Weis E: Chlorophyll Fluorescence and Photosynthesis: The Basics. Annual Review of Plant Physiology and Plant Molecular Biology 1991, 42(1):313-349.

42. Rochaix J-D: Assembly of the Photosynthetic Apparatus. Plant Physiology 2011, 155(4):1493.

43. Maxwell K, N Johnson G: Chlorophyll fluorescence-a practical guide, vol. 51; 2000.

44. King MC, Wilson AC: Evolution at two levels in humans and chimpanzees. Science (New York, NY) 1975, 188(4184):107-116.

45. Wittkopp PJ, Kalay G: Cis-regulatory elements: molecular mechanisms and evolutionary processes underlying divergence. Nature Reviews Genetics 2011, 13:59.

46. S Á, Sánchezblanco MJ: Comparison of individual and combined effects of salinity and deficit irrigation on physiological, nutritional and ornamental aspects of tolerance in Callistemon laevis plants. Journal of Plant Physiology 2015, 185:65-74.

47. Franco J, Martínez-Sánchez J, Fernández J, Bañón S: Selection and nursery production of ornamental plants for landscaping and xerogardening in semi-arid environments. The Journal of Horticultural Science and Biotechnology 2006, 81(1):3-17. 
48. Norton MR, Lelièvre tIF, Volaire F: Measuring dehydration tolerance in pasture grasses to improve drought survival. Crop and Pasture Science 2014, 65(8):828-840.

49. Sakuraba Y, Lee S-H, Kim Y-S, Park OK, Hörtensteiner S, Paek N-C: Delayed degradation of chlorophylls and photosynthetic proteins in Arabidopsis autophagy mutants during stress-induced leaf yellowing. Journal of Experimental Botany 2014, 65(14):3915-3925.

50. Sauter M, Moffatt B, Saechao MC, Hell R, Wirtz M: Methionine salvage and S-adenosylmethionine: essential links between sulfur, ethylene and polyamine biosynthesis. Biochem J 2013, 451(2):145-154.

51. Bitrian M, Zarza X, Altabella T, Tiburcio AF, Alcazar R: Polyamines under Abiotic Stress: Metabolic Crossroads and Hormonal Crosstalks in Plants. Metabolites 2012, 2(3):516-528.

52. Liang Y, Xiong Z, Zheng J, Xu D, Zhu Z, Xiang J, Gan J, Raboanatahiry N, Yin Y, Li M: Genome-wide identification, structural analysis and new insights into late embryogenesis abundant (LEA) gene family formation pattern in Brassica napus. Scientific Reports 2016, 6:24265.

53. Apelbaum A, Burgoon AC, Anderson JD, Lieberman M: Polyamines inhibit biosynthesis of ethylene in higher plant tissue and fruit protoplasts. Plant Physiol 1981, 68(2):453-456.

54. Suttle JC: Effect of polyamines on ethylene production. Phytochemistry 1981, 20(7):1477-1480.

55. Cheng MC, Liao PM, Kuo WW, Lin TP: The Arabidopsis ETHYLENE RESPONSE FACTOR1 regulates abiotic stress-responsive gene expression by binding to different cis-acting elements in response to different stress signals. Plant Physiol 2013, 162(3):1566-1582.

56. Smet ID, Zhang H, Inzé D, Beeckman T: A novel role for abscisic acid emerges from underground. Trends in Plant Science 2006, 11(9):434-439.

57. De SI, Signora L, Beeckman T, Inzé D, Foyer CH, Zhang H: An abscisic acid-sensitive checkpoint in lateral root development of Arabidopsis. Plant Journal 2003, 33(3):543-555.

58. Duan L, Dietrich D, Ng CH, Chan PM, Bhalerao R, Bennett MJ, Dinneny JR: Endodermal ABA signaling promotes lateral root quiescence during salt stress in Arabidopsis seedlings. Plant Cell 2013, 25(1):324.

59. Geng Y, Wu R, Wee CW, Xie F, Wei X, Chan PM, Tham C, Duan L, Dinneny JR: A spatio-temporal understanding of growth regulation during the salt stress response in Arabidopsis. Plant Cell 2013, 25(6):2132-2154.

60. Couée I, Hummel I, Sulmon C, Gouesbet G, Amrani AE: Involvement of polyamines in root development. Plant Cell Tissue \& Organ Culture 2004, 76(1):1-10.

61. Watson MB, Emory KK, Piatak RM, Malmberg RL: Arginine decarboxylase (polyamine synthesis) mutants of Arabidopsis thaliana exhibit altered root growth. The Plant Journal 1998, 13(2):231-239. 
62. Palavan-Ünsal N: Polyamine metabolism in the roots of Phaseolus vulgaris. Interaction of the inhibitors of polyamine biosynthesis with putrescine in growth and polyamine biosynthesis. Plant and cell physiology 1987, 28(4):565-572.

63. Kumar A, Taylor MA, Arif SA, Davies HV: Potato plants expressing antisense and sense Sadenosylmethionine decarboxylase (SAMDC) transgenes show altered levels of polyamines and ethylene: antisense plants display abnormal phenotypes. The Plant Journal 1996, 9(2):147-158.

64. Hattori T, Totsuka M, Hobo T, Kagaya Y, Yamamoto-Toyoda A: Experimentally Determined Sequence Requirement of ACGT-Containing Abscisic Acid Response Element. Plant and Cell Physiology 2002, 43(1):136-140.

65. Rouster J, Leah R, Mundy J, Cameron-Mills V: Identification of a methyl jasmonate-responsive region in the promoter of a lipoxygenase 1 gene expressed in barley grain. The Plant journal : for cell and molecular biology 1997, 11(3):513-523.

66. Krishnamurthy A, Rathinasabapathi B: Oxidative stress tolerance in plants: novel interplay between auxin and reactive oxygen species signaling. Plant Signal Behav 2013, 8(10):-.

67. Tognetti VB, MÜHlenbock PER, Van Breusegem F: Stress homeostasis - the redox and auxin perspective. Plant, Cell \& Environment 2012, 35(2):321-333.

68. Yamaguchi-Shinozaki K, Shinozaki K: Arabidopsis DNA encoding two desiccation-responsive rd29 genes. Plant Physiol 1993, 101(3):1119-1120.

69. Rushton PJ, Somssich IE, Ringler P, Shen QJ: WRKY transcription factors. Trends Plant Sci. Trends in Plant Science 2010, 15(5):247-258.

70. Petrov V, Vermeirssen V, De Cl, Van BF, Minkov I, Vandepoele K, Gechev TS: Identification of cisregulatory elements specific for different types of reactive oxygen species in Arabidopsis thaliana. Gene 2012, 499(1):52-60.

71. Wittkopp PJ, Kalay G: Cis-regulatory elements: molecular mechanisms and evolutionary processes underlying divergence. Nature Reviews Genetics 2012, 13(1):59-69.

72. Roberts MR, Foster GD, Blundell RP, Robinson SW, Kumar A, Draper J, Scott R: Gametophytic and sporophytic expression of an anther-specific Arabidopsis thaliana gene. The Plant journal : for cell and molecular biology 1993, 3(1):111-120.

\section{Figures' Legends}

Fig. 1 Comparative phylogenetic analysis of genes involved in polyamine and ethylene biosynthesis pathways. Red, blue and green dots represent genes participating in ethylene, polyamine and both (SAM 
family) pathways, respectively. The phylogenetic evolution of $C$. songorica proteins was determined using the known Arabidopsis and rice polyamine and ethylene pathway related protein sequences.

Fig.2 Expression profiles of $C$. songorica genes involved in polyamine and/or ethylene biosynthesis pathways under drought stress and rewatering treatments (a) and during salt stress, ABA, low temperature, and heat stress treatments $(b)$.

Fig. 3 qRT-PCR validation of expressions of different $C$. songorica genes involved in metabolic pathways genes. Expression of $C S A C C 3$, representative for ethylene pathway genes (a). Expression of the selected $S A M$ family gene (b) and expression of CSSAMDC2, a polyamine gene (c).

Fig. 4 Relative mRNA transcript expression level of CSSAMDC2 gene in plants upon $14 \mathrm{~d}$ of drought stress (a). Growth morphology of CSSAMDC2 lines and Col-0 under normal (b) and after $14 \mathrm{~d}$ of water withdraw (c).

Fig. 5 Morpho-physiology of transgenic Arabidopsis under normal watering conditions and after drought stress. Estimation of chlorophyll content in CSSAMDC2 Arabidopsis lines and in wild-type plants (a). PSII quantum efficiency ratio Fv/Fm of transgenic Arabidopsis lines overexpressing CSSAMDC2 genes compared to wild-type plants (b). The quantum efficiency of PSII was assayed during normal and after 14 $\mathrm{d}$ of drought stress. All plants were incubated under dark for 25 minutes before each measurement. The data are the means \pm standard errors. For each treatment, three biological samples were used and for each biological sample three technical replicates were used.

Fig. 6 Seedlings morphology was examined based on cotyledon greening rates plant architecture in transgenic and wild type plants grown under $150 \mathrm{mM} \mathrm{NaCl}$ stress conditions. After all plants reached the maximum germination on $150 \mathrm{mM} \mathrm{NaCl}$ stress medium (on the $28^{\text {th }}$ day ), they were transferred on a new medium of similar composition (a) to assess the germination percentage until the $45^{\text {th }}$ day(b).

Fig.7 Relative mRNA transcript expression level of polyamine genes AtADC2, AtASAMDC2, and ethylene genes AtACO2and AtACO6, and drought responsive genes AtRD29A and AtERF1 (a). Gene expression levels after $72 \mathrm{~h}$ of salt stress of polyamine genes (AtADC2 and AtASAMDC2), ethylene pathway genes (AtACO2 and AtACO6), a stress inducible gene AtRD29A, and of AtERF (ethylene responsive gene) (b). Effect of exogenous ABA on the expression of polyamine genes AtADC2 and AtASAMDC2, ethylene genes AtACO2 and AtACO6, stress inducible gene AtRD29A, and of an ethylene responsive gene, AtERF1(c). Expression levels were measured after $72 \mathrm{~h}$ and were relative to the expression before treatment $(0 \mathrm{~h})$ that was set to 1 . Data are the means \pm standard errors. For each treatment, three biological samples were used and for each biological sample three technical replicates were used.

Fig. 8 Effect of salt stress and ABA application on stress responsive gene activity and plant morphology. Morphology of wild type and transgenic plants under $150 \mathrm{mM} \mathrm{NaCl}$ at $0 \mathrm{~h}(\mathrm{a})$, after $72 \mathrm{~h}$ (b). The effect of ABA on root growth and morphology of CSSAMDC2 lines under $150 \mathrm{mM} \mathrm{NaCl}$ stress at $0 \mathrm{~h}$ (c) and after 72 $h(d)$. 
Fig. 9 Proposed network for stress response regulation by polyamine, ethylene pathway genes through SAM.

Additional file1 PCR Confirmation of Arabidopsis transgenic lines. PCR amplification of RD29A::CSSAMDC2 and 35S::CSSAMDC2 gene constructs (a). Amplification of 35S promoter (b) and RD29A promoter (c).

Additional file2: Supplementary table1, list of primers used in this study.

Additional file 3 Effects of CsSAMDC2 gene on germination under 0 and $100 \mathrm{mM} \mathrm{NaCl}$ stress of transgenic Arabidopsis plants. Photograph taken after 3 days following sowing on $0 \mathrm{mM} \mathrm{NaCl}$ medium (a). Photograph taken after 9 days after sowing on $100 \mathrm{mM} \mathrm{NaCl}$ medium(b). Germination percentage under $0 \mathrm{mM}$. d. Germination percentage on $100 \mathrm{mM} \mathrm{NaCl}$ stress (c). The data are the means \pm standard errors.

Additional file 4 Effect of CsSAMDC2 gene on germination under 150 and $200 \mathrm{mM} \mathrm{NaCl}$ stress of transgenic Arabidopsis plants. Photograph taken after 16 days following sowing on $150 \mathrm{mM} \mathrm{NaCl}$ medium(a) percent germination rates under $150 \mathrm{mM}(\mathrm{b})$. The data are the means \pm standard errors.

Additional file 5 Phenotyping transgenic and wild type Arabidopsis roots. Root length under $150 \mathrm{mMNaCl}$ salt stress(a) and under $150 \mathrm{mM} \mathrm{NaCl}+\mathrm{ABA}(\mathrm{b})$. For each treatment, three biological samples were used.

Additional file 6 Sequences of polyamine and ethylene proteins used in this study.

\section{Table}

Due to technical limitations, table 1 is only available as a download in the supplemental files section.

\section{Supplementary Files}

This is a list of supplementary files associated with this preprint. Click to download.

- Figure4ABC.tif

- Additionalfile3.tiff

- Figure8ABCD.tif

- Figure2AB.tif

- Figure5AB.tif

- Additionalfile5AB.tiff

- Figure1.tif

- Figure9.tif

- Figure6AandB.tif 
- Figure7abc.tif

- Additionalfile2.docx

- Table1.jpg

- Additionalfile6.docx

- Additionalfile1ABandC.tiff

- Figure3ABC.tif

- Additionalfile4.tiff 